\title{
In-Flight Performance Monitoring of TerraSAR-X Active Phased Array Antenna
}

\author{
Benjamin Bräutigam, Markus Bachmann, Marco Schwerdt \\ Microwaves and Radar Institute of the German Aerospace Center (DLR) \\ Oberpfaffenhofen, P.O.-Box 1116, D-82230 Weßling, Germany \\ Email: Benjamin.Braeutigam@dlr.de
}

\begin{abstract}
TerraSAR-X is a high resolution synthetic aperture radar (SAR) satellite launched in 2007. Its active phased array $\mathrm{X}$-Band antenna hosts 384 transmit/receive modules (TRMs) controlling the beam shape and steering in azimuth and elevation. A mathematical antenna model approach is used for pattern optimisation and calculation of all possible operation beams. For keeping the excellent radiometric performance of the SAR system the front-end antenna must be monitored throughout mission life time and calibrated if necessary. A calibration network provides monitoring of the instrument behaviour by evaluating internal calibration pulses. Evaluation of these calibration pulses for every image acquisition guarantees high radiometric stability of all SAR products. This paper shows the latest in-orbit results of the antenna performance and TRM stability complementary to the traditional approach of external antenna measurements over calibration targets. For this purpose, the novel PN Gating method is applied in a spaceborne environment for the first time ever.
\end{abstract}

\section{INTRODUCTION}

The first German SAR satellite for commercial and scientific applications, TerraSAR-X, is a flexible X-Band synthetic aperture radar (SAR) operating in Stripmap, Spotlight, ScanSAR, and additional experimental modes [1]. For the various antenna beams, an active phased array antenna electronically steers and shapes the patterns pattern in azimuth and elevation direction. The array consists of 384 radiating sub-arrays for horizontal and vertical polarisation arranged in a matrix of 12 panels with 32 rows each [2]. Active transmit/receive modules (TRMs) electronically excite the array elements in gain and phase [3]. An antenna model mathematically describes over 10,000 possible antenna patterns available for TerraSAR-X [4] using detailed on-ground measurements of the antenna geometry and its sub-arrays combined with the gain and phase excitations of the TRMs.

TerraSAR-X image products have an absolute radiometric accuracy of better than $0.6 \mathrm{~dB}$, based on the high relative radiometric accuracy and radiometric stability. This outstanding quality is achieved by external and internal calibration as well as establishing the accurate antenna model [5].

Temperature drifts and internal hardware characteristics cause instrument gain and phase fluctuation during data acquisition. TerraSAR-X hosts an internal calibration facility introducing internal calibration pulses into the front-end via a calibration network (see Fig. 1). Derived from these periodic gain and phase measurements instrument drifts can be calibrated to keep the required radiometric stability of $0.2 \mathrm{~dB}$.

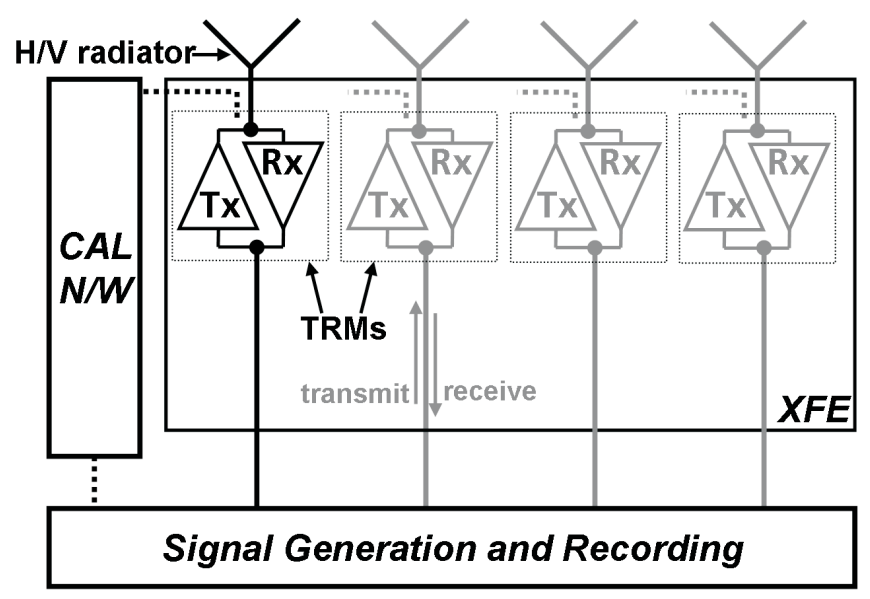

Fig. 1. X-Band Front-End (XFE) of TerraSAR-X Radar with 4 of 384 Transmit/Receive Modules (TRMs). The calibration signal is routed via couplers at the TRMs and the calibration network (CAL N/W).

Long-term performance degradation or failures of transmit/receive modules require permanent monitoring of the SAR front-end. Even though the X-band front-end (XFE) with a large number of TRMs is designed to be insensitive to contingencies like those of individually failed or drifted modules, it is necessary to detect such failures and characterise the TRMs.

In the module stepping mode of the ENVISAT ASAR instrument [6], individual measurements on the excitation coefficients of the TRMs are only possible if all modules except the one being characterised are switched off. However, representative measurements of TRMs require the same power loads like in the nominal mode with all TRMs operating. Hence, the efficient PN Gating method has been implemented into TerraSAR-X for a most realistic characterisation of the TRM performance [7]. Detected changes are fed into the antenna model for re-calculation of the actual antenna patterns.

A complete check of the antenna can be performed by external measurements over calibration targets. This covers all active and passive parts of the antenna. Using distributed targets like the Amazon rain forest as a homogeneous area, the antenna elevation pattern as well as the beam-to-beam gain offset can be measured against its calculated references from the antenna model. Both parameters allow only deviations of $0.2 \mathrm{~dB}$ to meet the specified relative radiometric accuracy. 
This paper provides an overview on the antenna performance monitoring and instrument calibration activities of TerraSAR-X, which are the:

- Internal Instrument Calibration to compensate the radar instrument drift,

- Individual TRM Performance Monitoring by using the novel PN Gating method,

- Antenna Pattern Monitoring by comparing external measurements to the antenna model reference.

For the first time, in-orbit measurements of TerraSAR-X long-term antenna performance are presented. The different monitoring and calibration activities are compared to each other with respect to the first year of satellite operation. The excellent results of the described monitoring methods promise a high SAR image quality throughout mission life time.

\section{INTERNAL INSTRUMENT CALIBRATION}

\section{A. Radiometric Stability}

TerraSAR-X features an internal calibration network coupling into an additional port of each TRM as shown in Fig. 1. Calibration pulses are routed through the XFE to characterise critical elements of radar path. The acquired signals can only be measured at the composite ports of the distribution networks.

Evaluating the amplitude and phase of the calibration signals provides information how to model the instrument drift during data acquisition. Thermal fluctuations as well as internal switching cause variations in gain and phase of the front-end components. This drift is corrected during image processing to obtain the high-quality of TerraSAR-X image products.

During the first in-orbit check of the internal calibration facility the SAR instrument was operated in a test mode replacing all imaging pulses with calibration pulses. By these means, the actual instrument drift can be extracted from calibration pulses.

Fig. 2 shows the influence of the instrument on the real radar data over time. For a time frame of one minute, the gain drift is about $0.4 \mathrm{~dB}$ and the phase drift is less than 6 degrees during nominal SAR operation showing a very stable radar instrument. Applying calibration sequences before, inbetween, and after acquisition, the instrument drift can be accurately determined and calibrated. The residual calibration error is very small resulting in less than $0.1 \mathrm{~dB}$ in gain and less than $1^{\circ}$ in phase [8].

\section{B. Instrument Gain Monitoring}

In ground processing the replica for range compression is directly calculated from the calibration pulses. This ensures a higher correlation during pulse compression compared to a range reference function derived from ideal waveform parameters. Additionally, the replica amplitude behaviour can be transformed into instrument gain. The SAR image is processed with this gain value at the time of data taking. Thus, the actual instrument gain status is calibrated during ground processing.



Fig. 2. Drift of instrument gain (upper figure) and phase (lower figure) over time in blue. The black curve shows calibration points during acquisition. The green line is a linear fit from start to end calibration sequence.

The replica gain variation over mission time is an indicator for the stability of the instrument. Fig. 3 shows the mean amplitude of all replicas used since start of the operational phase for range compression of the respective data takes. The mean amplitude is very constant even one year after launch. The fluctuations in the order of $\pm 1 \mathrm{~dB}$ are caused by temperature variations of the front end, as shown in Fig. 4. Still, these variations can be compensated for by applying the replica mean amplitude in the image range compression.

For comparison, the daily measured temperature of the antenna heat capacity blocks (close to the front-end TRMs) is plotted over the same time. The temperature variation is due to the operational load on-board of the satellite. The frontend gets warmer with higher instrument usage per orbit. This explains the variations of the replica mean amplitude.

\section{INDIVIDUAL TRM PERFORMANCE MONITORING}

Apart from measuring the stability of the instrument gain it is necessary to retrieve information on the performance status of individual transmit/receive modules (TRMs). Tapering and steering of the antenna beam depends on the beam excitation coefficients defining gain and phase of the TRMs. The actual status of the TRM settings has to be known, especially considering performance degradation or malfunction. The actual settings are fed into the antenna model to derive the real antenna patterns [4], [5].

The detailed analysis of individual TRMs within an active phased array antenna is based on the so-called PN Gating method developed at the German Aerospace Center (DLR) [7]. Compared to the module stepping mode of ENVISAT ASAR the advantage of this technique is that individual TRMs are characterised while all modules are operating, i.e. a characterisation under the most realistic conditions. 




Fig. 3. Mean Amplitude of Range Reference Chirp over time since start of the operational phase of TerraSAR-X.

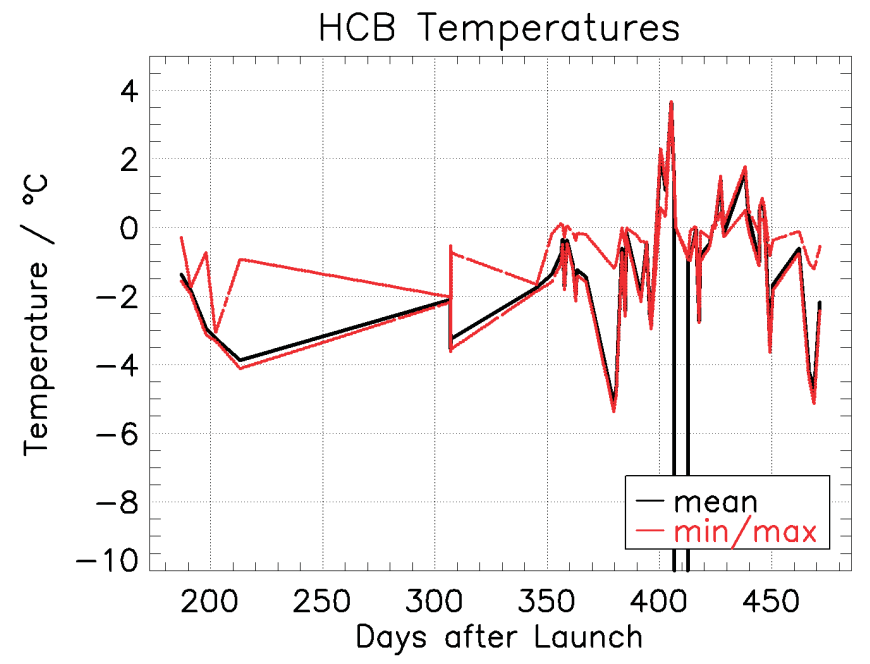

Fig. 4. Daily temperature of front-end heat capacity blocks over time. The low temperature during day $400(23.07 .2008)$ is due to a telemetry data gap.

In this special characterisation mode the actual phase of each TRM is individually shifted by $\pm 90^{\circ}$ degrees from pulse to pulse according to a defined code sequence. Consequently, the superposition of all TRM gains and phases at the composite port of the distribution network yields the composite signal. To extract the information for one TRM this composite signal is correlated with the corresponding code sequence providing the estimated gain and phase setting of this module.

Since start of the operational phase 60 TRM evaluations have been done with the PN Gating method. Each TRM is characterised for gain and phase in transmit and receive case. This gives a comprehensive view on the TRM performance over time. Fig. 5 and Fig. 6 present the TRMs' transmit longterm performance. They show a stable behaviour over time with no TRM degradation or failure since launch. There is no

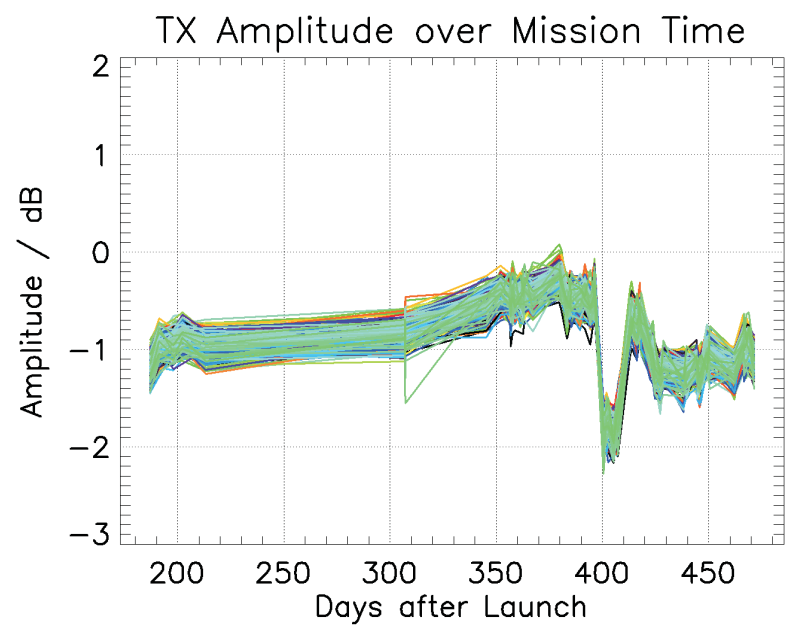

Fig. 5. Measurement variation of all TRMs transmit gains over time since start of the operational phase. The individual measurements are referenced to the respective average value determined during the satellite commissioning phase.

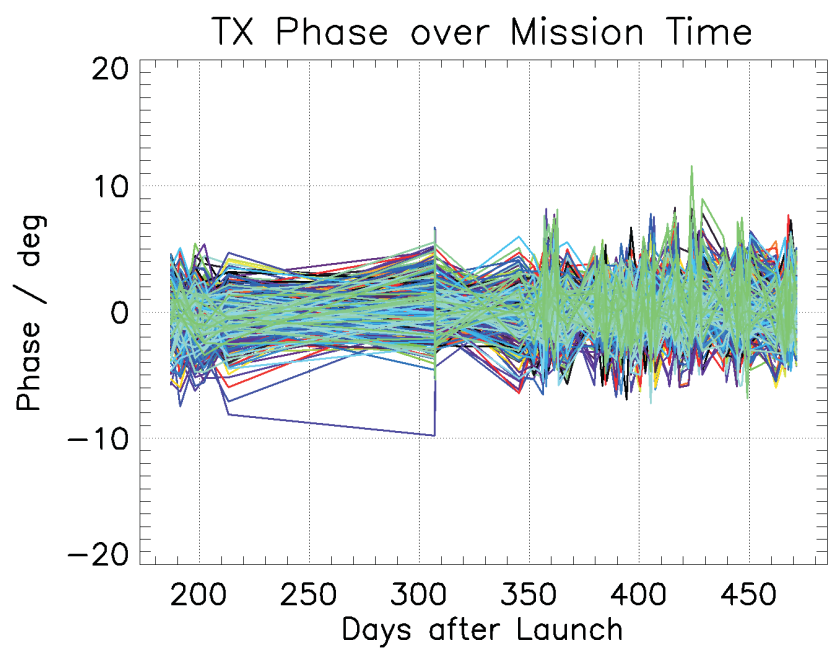

Fig. 6. Measurement variation of all TRMs transmit phases over time since start of the operational phase. The individual measurements are referenced to the respective average value determined during the satellite commissioning phase.

trend for strong deviations compared to the reference values.

The TRMs' total gain variation over time is due to the different temperature conditions in the instrument (compare Fig. 3 and Fig. 4). As this variation of the total instrument gain is likewise extracted from the calibration pulses and compensated for during ground processing, the system stays absolutely calibrated, as shown in chapter II-B.

The above results on individual TRM performance prove the high stability of the TerraSAR-X active antenna of better than $0.2 \mathrm{~dB}(\mathrm{rms})$ in gain and $2^{\circ}(\mathrm{rms})$ in phase. These measurements also verify the successful implementation of the PN Gating method in a spaceborne environment for the first time ever. 


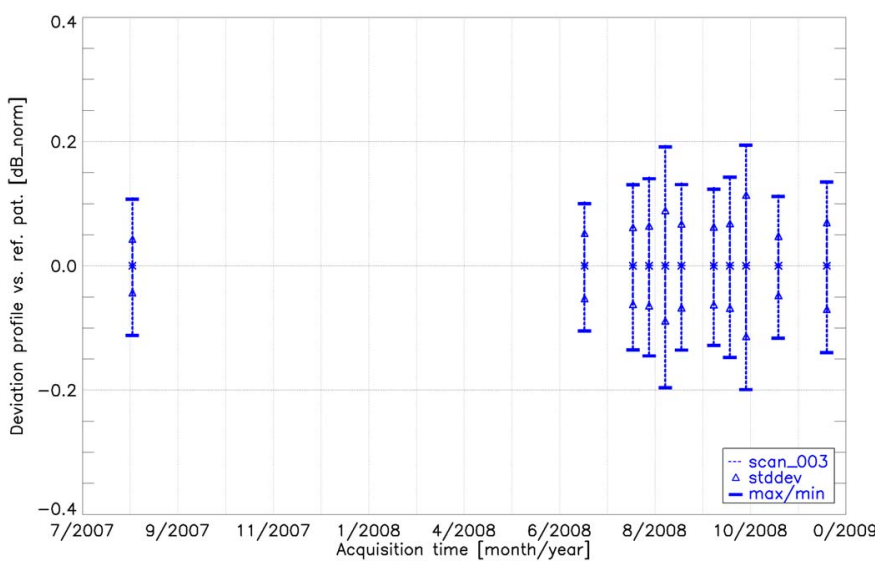

Fig. 7. Relative deviation of the antenna patterns measured for long-term system monitoring from the reference antenna patterns.

\section{Antenna Pattern Monitoring}

To ensure a constant product quality and accurately calibrated images, the SAR antenna patterns are monitored on a regular basis, too. The antenna patterns are mainly needed for SAR image correction. The long-term monitoring shall detect changes or degradation in the passive or active parts of the antenna. For the purpose of regular image acquisitions a test site in the Amazon rain forest has been established. Furthermore, a set of four beams in ScanSAR operation has been selected to enable monitoring of both the pattern shape as well as the antenna model beam-to-beam gain offset prediction. From the acquisitions, three different parameters can be derived in order to evaluate the performance of the antenna patterns:

- the mean value of the deviation between the measurement and the reference pattern,

- the corresponding standard deviation for each pattern, and

- the minimum and maximum deviation within the complete set of the four beams.

During the TerraSAR-X commissioning phase [5], a verification of the antenna model was performed. Here, the ability of the antenna model to derive the antenna patterns with an accuracy of $\pm 0.2 \mathrm{~dB}$ was proven for both the patterns shape and the beam-to-beam gain prediction. The actual relative deviation of measured patterns from the reference patterns is within the requirement of $\pm 0.2 \mathrm{~dB}$ for both the shape within the main beam and the gain offset between these beams. There is no trend for degradation over mission time. This is shown in Fig. 7 with each blue line describing one measurement and its antenna pattern performance parameters, respectively.

\section{CONCLUSION}

Synthetic aperture radars (SAR) with active phased array antennas depend on the reliable operation of its electronic instrument components. For the TerraSAR-X satellite, the stability of its 384 transmit/receive modules (TRMs) is of crucial importance for fast and flexible generation of various antenna beams. Covering over 10,000 antenna beams in different SAR operation modes, periodic performance monitoring must be repeated throughout mission lifetime to keep the high product quality of TerraSAR-X. In case of degradations or failures the system is capable of dynamic re-calibration using the antenna model with input from internal calibration measurements.

Besides nominal calibration with calibration pulses, the internal calibration network provides direct monitoring of the radar instrument performance. The instrument drift can be derived and compensated with a calibration accuracy of better than $0.1 \mathrm{~dB}$ for gain and $1^{\circ}$ in phase. The long-term instrument gain variation is very small and stays constant over mission time. Still, this variation is calibrated in the ground processing.

The presented TRM characterisation method based on the novel PN Gating technique has become a valuable diagnostic tool during satellite operation for fast and realistic TRM performance checks. The results from repeated in-orbit measurements prove the high reliability of the PN Gating method better than $0.2 \mathrm{~dB}$ for amplitude and $2^{\circ}$ for phase estimation.

The antenna model updates the actual antenna patterns derived from individual TRM measurements by PN Gating. External antenna measurements over rain forest provide monitoring of both the pattern shape as well as the beamto-beam gain offset calculated in the antenna model. The evaluation of antenna patterns monitored across the rainforest shows likewise a very high stability of $\pm 0.2 \mathrm{~dB}$ of the whole TerraSAR-X system.

The calibration techniques described in this paper are an essential contribution to in-orbit monitoring of the performance of active phased array antennas and can be applied to future SAR systems like TanDEM-X [9], too.

\section{REFERENCES}

[1] S. Buckreuss, W. Balzer, P. Mühlbauer, R. Werninghaus, and W. Pitz, "The TerraSAR-X Satellite Project," in IEEE: 25th International Geoscience And Remote Sensing Symposium, Toulouse, Frankreich, 2003.

[2] W. Pitz, "The TerraSAR-X Satellite," in 6th European Conference on Synthetic Aperture Radar, Dresden, Germany, 2006.

[3] M. Wahl, M. Adolph, K. Biller, U. Hackenberg, R. Rieger, B. Schweizer, B. Adelseck, H. Brugger, and M. Loercher, "High Precision T/R Module for SAR Earth Observation," in 6th European Conference on Synthetic Aperture Radar, Dresden, Germany, 2006.

[4] M. Bachmann, M. Schwerdt, B. Bräutigam, B. Grafmüller, A. Herschlein, and J. L. Alvarez-Pérez, "The TerraSAR-X Antenna Model Approach," in 2nd International ITG Conference on Antennas, Munich, Germany, 2007.

[5] M. Schwerdt, B. Bräutigam, M. Bachmann, and B. Döring, "Final Results of the Efficient TerraSAR-X Calibration Method," in IEEE Radar Conference, Rome, Italy, 2008.

[6] C. Buck, "Asar instrument gain monitoring," ESTEC, European Space Agency (ESA), ENVISAT ASAR Calibration Review (ECR), 2002.

[7] B. Bräutigam, M. Schwerdt, M. Bachmann, and M. Stangl, "Individual T/R Module Characterisation of the TerraSAR-X Active Phased Array Antenna by Calibration Pulse Sequences with Orthogonal Codes," in IEEE: 27th International Geoscience And Remote Sensing Symposium, Barcelona, Spain, 2007.

[8] B. Bräutigam, J. Hueso, M. Schwerdt, and M. Bachmann, "Radar Instrument Calibration of TerraSAR-X," in 7th European Conference on Synthetic Aperture Radar, Friedrichshafen, Germany, 2008.

[9] G. Krieger, A. Moreira, H. Fiedler, I. Hajnsek, M. Werner, M. Younis, and M. Zink, "TanDEM-X: A Satellite Formation for High Resolution SAR Interferometry," IEEE Transactions on Geoscience and Remote Sensing, pp. 206-218, November 2007. 